\title{
Comparing Touchscreen and Mouse Input Performance by People With and Without Upper Body Motor Impairments
}

\author{
Leah Findlater' ${ }^{1}$, Karyn Moffatt ${ }^{3}$, Jon E. Froehlich ${ }^{2}$, Meethu Malu' ${ }^{2}$, Joan Zhang ${ }^{2}$ \\ ${ }^{1,2}$ Human-Computer Interaction Lab \\ ${ }^{1}$ College of Information Studies | ${ }^{2}$ Dept. of Computer Science \\ ${ }^{3}$ ACT Research Group \\ University of Maryland, College Park, MD \\ leahkf@umd.edu, \{jonf, meethu\}@cs.umd.edu, joan.r.zhang@gmail.com \\ School of Information Studies \\ McGill University, Montreal, QC \\ karyn.moffatt@mcgill.ca
}

\begin{abstract}
Controlled studies of touchscreen input performance for users with upper body motor impairments remain relatively sparse. To address this gap, we present a controlled lab study of mouse $v s$. touchscreen performance with 32 participants (16 with upper body motor impairments and 16 without). Our study examines: (1) how touch input compares to an indirect pointing device (a mouse); (2) how performance compares across a range of standard interaction techniques; and (3) how these answers differ for users with and without motor impairments. While the touchscreen was faster than the mouse overall, only participants without motor impairments benefited from a lower error rate on the touchscreen. Indeed, participants with motor impairments had a three-fold increase in pointing (tapping) errors on the touchscreen compared to the mouse. Our findings also highlight the high frequency of spurious touches for users with motor impairments and update past accessibility recommendations for minimum touchscreen target sizes to at least $18 \mathrm{~mm}$.
\end{abstract}

\section{Author Keywords}

Human performance; motor impairments; accessibility; input devices; touchscreen; mouse.

\section{ACM Classification Keywords}

H.5.m. Information interfaces and presentation (e.g., HCI): Miscellaneous; K.4.2. Social issues: assistive technologies for persons with disabilities.

\section{INTRODUCTION}

Mobile devices can increase independence and provide a sense of empowerment for people with motor impairments $[1,7,11]$. While the shift to touchscreens has provided some benefits to motor-impaired users, such as requiring little force to register a tap and offering the flexibility to use alternative body parts (e.g., a knuckle) [16], many accessibility challenges exist, ranging from simply pulling out the device [11] to being able to tap precisely without slipping [16].

Permission to make digital or hard copies of all or part of this work for personal or classroom use is granted without fee provided that copies are not made or distributed for profit or commercial advantage and that copies bear this notice and the full citation on the first page. Copyrights for components of this work owned by others than ACM must be honored. Abstracting with credit is permitted. To copy otherwise, or republish, to post on servers or to redistribute to lists, requires prior specific permission and/or a fee. Request permissions from Permissions@acm.org. CHI 2017, May 06-11, 2017, Denver, CO, USA

(C) 2017 ACM. ISBN 978-1-4503-4655-9/17/05 ..\$15.00 DOI: http://dx.doi.org/10.1145/3025453.3025603

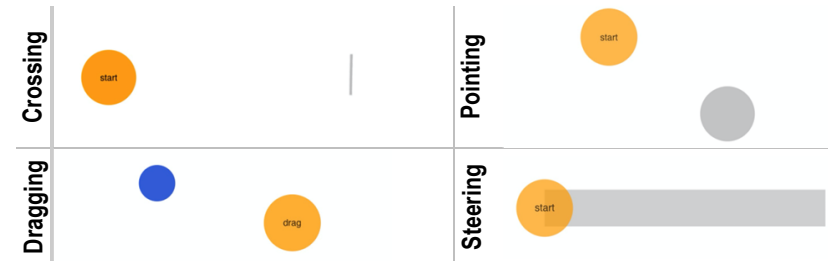

Figure 1. Cropped screenshots of the four study tasks, showing a range of orientations, target widths, and amplitudes.

Despite the above body of work, controlled studies of touchscreen input performance for people with upper body motor impairments are relatively sparse. Studies have compared novel techniques (e.g., swabbing [17]) to a control condition such as tapping $[9,10,17,20]$, but do not shed light on more general questions related to device comparisons (e.g., touchscreen vs. mouse) and user groups. Other studies have compared tapping input performance for users with and without motor impairments, but using vertical touchscreen kiosks (e.g., [6,14]), where the large target size and device orientation do not directly translate to mobile touchscreens.

The most comprehensive evaluations come from Trewin et $a l$. [16] and Guerreiro and colleagues [3,12]. The former [16] evaluates a range of native interaction techniques within existing mobile apps, which is useful for ecological validity but did not allow for precise quantification of performance. The latter [3,12] provides a more precise measurement of speed and accuracy for users with and without motor impairments across common interactions (pointing, crossing, directional swiping), separately analyzing the data for each task and each group. However, the lack of direct comparisons and the use of non-standard input tasks (e.g., as opposed to [5]) leads to an incomplete understanding of the inherent tradeoffs of different touchscreen interaction techniques for users with and without motor impairments. Neither evaluation included a mouse comparison.

Addressing these gaps, we conducted a controlled lab study to understand: (1) how touch input compares to indirect pointing devices (e.g., a mouse); (2) how input performance compares across a range of standard interaction techniques; (3) how these answers differ for users with and without motor impairments. Thirty-two participants (16 with and 16 without motor impairments) completed a set of basic input tasks with both a mouse and a touchscreen tablet: crossing, dragging, pointing, and steering. We found a high error rate for touchscreen pointing (tapping) compared to mouse pointing for users with motor impairments, and, relatedly, a 
disparity in how direct touch benefits users with and without motor impairments. Secondarily, we provide a more detailed comparison of users with and without motor impairments in terms of two facets of touchscreen input accuracy: the impacts of target size and spurious touch frequency.

\section{METHOD}

Our method was largely consistent with past work [2].

\section{Participants}

This study included 16 participants with upper body motor impairments (WithMI) and 16 without (NoMI). The WithMI group was on average 42.0 years old $(S D=16.8)$, with 11 females and 5 males. They reported using computers daily $(N=9)$, a few times a week (5), or a few times a month (2), and using touchscreen devices daily $(N=13)$, a few times a week (1) or never (2). More detail on the WithMI group is in the Supplemental Materials, but reported medical conditions included cerebral palsy $(N=5)$, multiple sclerosis (4), C5 spinal cord injury (1), essential and orthostatic tremor (1), sports injury (1), arthritis (1), stroke (1), and traumatic brain injury (1). ${ }^{1}$ The NoMI group was on average 19.8 years old $(S D=1.1)$, with 10 females and 6 males; all reported daily use of computers and touchscreen devices. ${ }^{2}$

\section{Apparatus}

The experimental testbed was written in JavaScript, HTML, and PHP. For the mouse condition, the testbed was loaded in a Chrome browser on a Mac laptop running OS $\mathrm{X}$ and connected to an external monitor with $1280 \times 1024$ resolution and a Logitech M310 wireless optical mouse. For the touchscreen, the testbed was loaded in Safari on an Apple iPad 3 in portrait orientation. The testbed guided participants through the four input tasks (crossing, steering, pointing, and dragging), each implemented based on the ISO 9241-9 circle 2D Fitts' law task standard [5]. We fully crossed amplitudes $(A)$ of $\{250 \mathrm{px}, 500 \mathrm{px}\}$ with widths $(W)$ of $\{32 \mathrm{px}, 64 \mathrm{px}$, $96 \mathrm{px}\}$, removing the combination $(A=500 \mathrm{px}, W=96 \mathrm{px})$ as it did not fit on the iPad screen with enough padding to allow a $2 \mathrm{~W}$ overshoot of the target (the iPad is only 768px wide and the task canvas had to be square for the ISO task). The five $A \times W$ combinations thus provide an Index of Difficulty (ID) range of 1.9-4.1, where $I D$ is the ratio between the distance to the target and its width and where higher values indicate greater input difficulty [15]: ID $=\log _{2}(\mathrm{~A} / \mathrm{W}+1)$. On the $\mathrm{iPad}$, the smallest target width, $32 \mathrm{px}$, corresponds to a $6 \mathrm{~mm}$ target.

\section{Procedure}

The procedure took up to one hour for the NoMI group and 90 minutes for the WithMI group, but otherwise was exactly the same for both. The iPad was placed flat on the table, but participants could adjust its specific location. Mouse speed was set to the OS X default. Participants could raise/lower the table before starting, necessary for some wheelchair users. The mouse and touchscreen were presented in

\footnotetext{
${ }^{1}$ One participant's response was unclear.

2 The NoMI data was collected, in part, for a different study with 30 participants. We randomly selected 16 of these 30 NoMI participants to ensure equal sample sizes with the WithMI group.
}

counterbalanced order, with the crossing, dragging, pointing, and steering tasks randomly ordered for each device. ${ }^{3}$ For each task, participants completed 5 practice trials (which they could opt to repeat once) and 55 test trials. Each trial began by activating a circular start target by tapping/clicking (pointing and dragging tasks) or holding/hovering for $300 \mathrm{~ms}$ (crossing and steering tasks); see Figure 1. Spatial outlier trials, that is, where the movement distance was less than half of $A$ or the endpoint was more than $2 W$ away from the target center [8], were automatically redone by appending them to the end of the set of trials. Short breaks were offered midway through each task, between tasks, and mid-session. Background information was collected between tasks.

\section{Experiment Design, Data, and Analysis}

We used a $2 \times 2 \times 4$ mixed factorial design: Group (WithMI $v s$. NoMI) $\times$ Device (mouse vs. touchscreen) $\times$ Task (crossing, dragging, pointing, steering). Order of presentation for Device was counterbalanced, while Task was randomized. As mentioned above, participants repeated spatial outlier trials, consistent with prior work [8,19]. Doing so assumes, however, that a spatial outlier is spurious in some wayperhaps an accidental touch or lift off when the participant is momentarily distracted. While this assumption may be reasonable for users without motor impairments [8,19], our WithMI group had a relatively high incidence of spatial outliers: $4.5 \%$ of trials vs. only $0.5 \%$ for the NoMI group. As such, we consider these trials to be valid; for both groups, we ignore the appended trials and only analyze the first attempt of each trial. Our dataset includes 14080 trials in total.

Our primary speed and error rate analysis includes all three factors in our experimental design. We use parametric tests (e.g., ANOVAs, t-tests) for speed, and non-parametric alternatives (e.g., Aligned Rank Transform ANOVAs [18], Wilcoxon signed ranks tests) for most other measures as they did not meet the normality assumption of parametric tests. When the degrees of freedom are fractional for ANOVAs, a Greenhouse-Geisser adjustment has been applied to account for sphericity violations. Posthoc pairwise comparisons were protected against Type I error using a Bonferroni adjustment.

\section{RESULTS}

We first compare the touchscreen and mouse before focusing specifically on issues with touchscreen accuracy.

\section{Touchscreen versus Mouse}

Speed. Overall speed results are shown in Figure 2, with detailed ANOVA results in Table 1. As expected, all three main effects (Group, Device, and Task) were significant: the WithMI group was slower than the NoMI group, the touchscreen was faster than the mouse, and tasks impacted speed differently. There were also significant interaction effects of Group $\times$ Task and Device $\times$ Task. Focusing on the interaction effect involving Group, our primary factor of

\footnotetext{
${ }^{3}$ On the iPad, these primary tasks were optionally followed by pinch and zoom, which only half of the WithMI group completed due to ability and/or time. Thus, we do not report on pinch/zoom.
} 


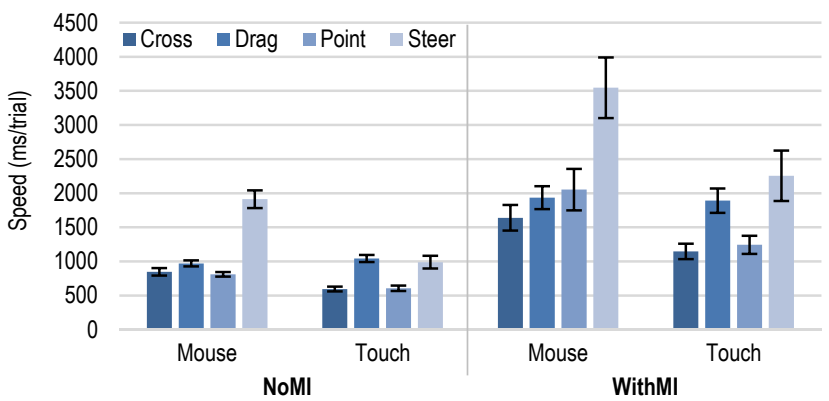

Figure 2. Average trial speed for participant groups without and with motor impairments $(N=32$; error bars show standard error). Lower values are better.

\begin{tabular}{l|lll} 
Effect & Result & & \\
\hline Group & $F_{1,30}=29.91$ & $p<.001$ & partial $\eta^{2}=.50$ \\
Device & $F_{1,30}=25.12$ & $p<.001$ & partial $\eta^{2}=.46$ \\
Task & $F_{1.83,54.94}=29.91$ & $p<.001$ & partial $\eta^{2}=.56$ \\
Group $\times$ Device & $F_{1,30}=2.85$ & $p=.102$ & partial $\eta^{2}=.09$ \\
Group $\times$ Task & $F_{1.83,54.94}=4.14$ & $p=.024$ & partial $\eta^{2}=.12$ \\
Device $\times$ Task & $F_{1.55,46.58}=14.00$ & $p<.001$ & partial $\eta^{2}=.32$ \\
Group $\times$ Device $\times$ Task & $F_{1.55,46.58}=0.70$ & $p=.467$ & partial $\eta^{2}=.02$ \\
Table 1. Three-way (Group $\times$ Device $\times$ Task) repeated measures \\
ANOVA results for speed.
\end{tabular}

interest, we conducted posthoc pairwise comparisons of each of the four tasks within each group. For the WithMI group, steering fared the worst, at $2,901 \mathrm{~ms}(S D=1,301)$ per trial; it was significantly slower than all three other tasks (all $p<$ $.05)$. Dragging was also relatively slow, being significantly slower than crossing $(p<.05)$. For the NoMI group, steering was significantly slower than crossing and pointing (all $p<$ $.05)$, but not different from dragging.

Overall, the relative performance of the four tasks was largely similar across the two groups for speed. For the most part, the WithMI group was simply slower, by on average about double the time. This is the case for both devices and all four tasks, with the possible exception of dragging versus crossing for the WithMI group.

Errors. The error rate results (Figure 3) are more complex. While Table 2 includes all detailed results from the ANOVA with ART, here we focus on the main effect of Group and interaction effects involving Group $\times$ Device, our primary and secondary factors of interest.

The NoMI group's average error rate was $6.1 \%(S D=4.3)$ with the mouse and $3.2 \%(S D=2.2)$ with the touchscreen. In contrast, the WithMI group had a significantly higher error rate, at $21.1 \%(S D=19.7)$ with the mouse and $25.1 \%(S D=$ 21.0) with the touchscreen - in the latter case, almost eight times as high as the NoMI group. These patterns translated into a significant main effect of Group and a significant Group $\times$ Device interaction. Based on the interaction, posthoc pairwise comparisons using Wilcoxon signed ranks tests showed that the touchscreen resulted in significantly lower error rates than the mouse for the NoMI group ( $p<$ $.05)$; no similar benefit was seen for the WithMI group.

Finally, there was a significant Group $\times$ Device $\times$ Task interaction. Pairwise comparisons showed that for NoMI participants, crossing and steering were more accurate with

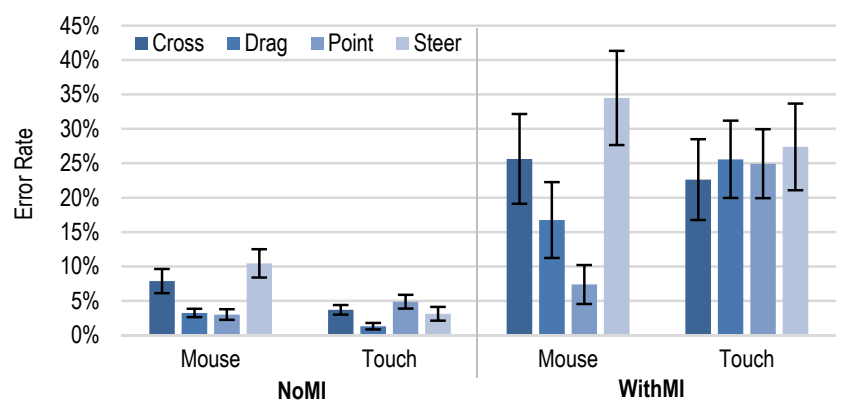

Figure 3. Average error rate for participant groups without and with motor impairments $(N=32$; error bars show standard error). Lower values are better.

\begin{tabular}{c|lll} 
Effect & Result & & \\
\hline Group & $F_{1,30}=14.81$ & $p=.001$ & partial $\eta^{2}=.33$ \\
Device & $F_{1,30}=3.11$ & $p<.001$ & partial $\eta^{2}=.09$ \\
\hline Task & $F_{3,90}=22.03$ & $p<.001$ & partial $\eta^{2}=.42$ \\
Group $\times$ Device & $F_{1,30}=22.70$ & $p<.001$ & partial $\eta^{2}=.43$ \\
Group $\times$ Task & $F_{3,90}=11.00$ & $p<.001$ & partial $\eta^{2}=.27$ \\
Device $\times$ Task & $F_{2.35,70.56}=28.98$ & $p<.001$ & partial $\eta^{2}=.49$ \\
Group $\times$ Device $\times$ Task & $F_{2.29,68.63}=9.79$ & $p<.001$ & partial $\eta^{2}=.25$ \\
\multicolumn{1}{c}{ Table 2. Three-way $($ Group $\times$ Device $\times$ Task) repeated } \\
measures ANOVA with ART results for error rate.
\end{tabular}

the touchscreen than the mouse $(p<.05)$, but no differences were found between the two devices for dragging or pointing. For WithMI participants, in comparison, pointing was the only task with significant differences between the two devices $(p<.05)$. Here, the error rate was $24.9 \%(S D=$ $20.0)$ with the touchscreen versus only $7.4 \%(S D=11.3)$ with the mouse. This finding has important practical implications for accessibility as pointing (tapping) is by far the most common touchscreen interaction technique.

\section{A Closer Look at Touchscreen Errors}

Following the disparity in how the touchscreen impacted errors for the two user groups, we conducted more detailed error analyses to examine target size and spurious touches.

Target size. Past work has recommended $12 \mathrm{~mm}$ as an appropriate touchscreen target size for supporting users both with and without motor impairments based on seeing no further error reduction at larger sizes [12]. To revisit this finding and to directly compare the effects of target size for the NoMI and WithMI groups (unlike [12]'s separate analyses), we analyzed error rate based on the three target sizes in our task: $6 \mathrm{~mm}, 12 \mathrm{~mm}$, and $18 \mathrm{~mm}$. Figure 4 shows the overall results. A $2 \times 3$ (Group $\times$ Target Size) ANOVA with ART showed that there were significant main effects of both Group $\left(F_{1,30}=35.76, p<.001, \eta_{p}^{2}=.54\right)$ and Target Size $\left(F_{1.48,60}=67.16, p<.001, \eta_{p}^{2}=.69\right)$ on average error rate.

More interesting, however, was a significant Group $\times$ Target Size interaction effect, showing that target size impacted the WithMI group differently than the NoMI group $\left(F_{1.40,60}=\right.$ $\left.67.16, p<.001, \eta_{p}^{2}=.58\right)$. The WithMI group had an average error rate of $42.1 \%(S D=29.2)$ with the smallest target size $(6 \mathrm{~mm})$, which dropped to $7.0 \%(S D=11.3)$ for the largest size $(18 \mathrm{~mm})$. For NoMI participants, error rates were close to zero for the larger two target sizes, but jumped to $7.4 \%$ on average $(S D=4.7)$ for the smallest targets-comparable to 


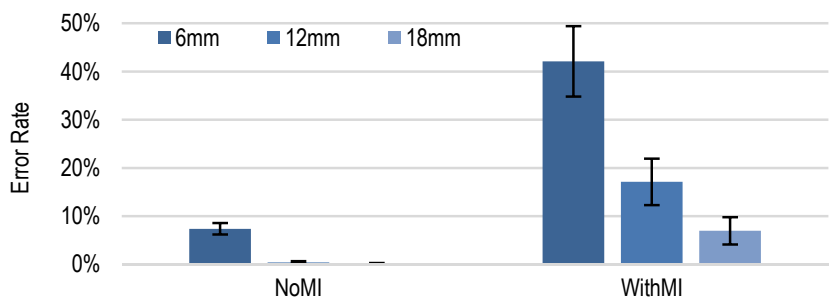

Figure 4. Touchscreen error rates by target size, collapsed across all four tasks ( $N=32$; error bars show standard error).
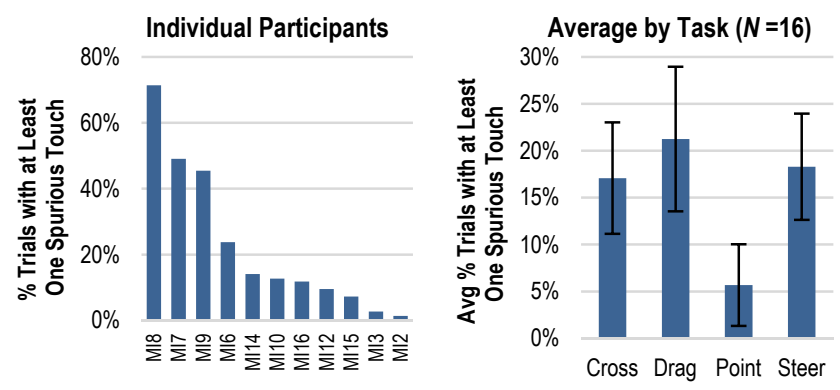

Figure 5. Spurious touches for the WithMI group (the NoMI group made almost no spurious touches): by participant (left) and by task (right). MI1, MI4, MI5, MI11 and MI13 made no spurious touches, thus are not shown in the individual chart.

the largest target size for WithMI participants. Pairwise comparisons based on the interaction effect emphasize these results: for the WithMI group, the smallest targets caused significantly more errors than the other two sizes, and the medium targets caused more errors than the largest ones (all $p<.05)$. For the NoMI group, the smallest targets caused significantly more errors than the other two sizes $(p<.05)$; there was no difference between the medium and large targets. The NoMI results are consistent with past work [4] and a recommended target size of $\sim 9 \mathrm{~mm}[13]$.

Spurious touches. While spurious touches are ignored in typical Fitts task (as in our earlier analysis), they can create problems in real systems, for example, causing an intended tap to result in a multi-finger gesture like zoom. While Mott et al. [10] report on the number of touchpoints on the screen during tapping tasks, participants in that study tapped in a "comfortable and natural" way, rather than under standard conditions. To our knowledge, spurious touches have not been reported in detail for users with motor impairments. Thus, we logged spurious touches for posthoc analysis.

Spurious touch rates for the WithMI group are shown in Figure 5. For this group, on average $5.7 \%$ of pointing trials $(S D=17.4)$ had one or more spurious touches, rising to $21.3 \%(S D=30.8)$ for dragging trials. Spurious touches were also much more of a problem for some WithMI participants than others. Five of the 16 participants made no spurious touches at all, while participant MI8 made at least one spurious touch in $71 \%$ of trials. For comparison, only two NoMI participants made any spurious touches, for a total of four touches that all occurred in the dragging task.

Due to the lack of variance in the data from the NoMI group, we did not conduct a factorial analysis comparing the two groups. However, a Friedman test with the single factor of
Task for the WithMI group was statistically significant $\left(\chi_{3, \mathrm{~N}=16}^{2}=15.84, p<.001\right)$. This result is not surprising, given that crossing, dragging, and steering require continuous touching during the trial, therefore increasing the chance of additional spurious touches compared to pointing.

\section{DISCUSSION AND CONCLUSION}

While our study shows that there is an overall speed advantage to the touchscreen over the mouse, touchscreen error rates were only lower than the mouse for users without motor impairments. Indeed, for users with motor impairments, touchscreen tapping (i.e., pointing) errors were more than three times as high with the touchscreen than with the mouse. Tapping is by far the most common touchscreen interaction technique, which emphasizes the importance of efforts to improve tapping accuracy (e.g., [10,20]).

Our results also update Nicolau et al.'s [12] recommended minimum touchscreen target size for users with upper body motor impairments. Their suggestion of $12 \mathrm{~mm}$ was based on seeing no improvement from $12 \mathrm{~mm}$ to $17 \mathrm{~mm}$, but error rates were over $20 \%$ for both sizes - likely unacceptably high for real use and potentially due to their participants being much less experienced with touchscreens than ours. Our results suggest that touchscreen targets should be at least $18 \mathrm{~mm}$ to be accessible for users with a range of upper body motor impairments. Even at $18 \mathrm{~mm}$, our error rate was $7 \%$, so future work should examine at what point the rates level off.

Nicolau et al. [12] also concluded that swipes were inaccessible for users with motor impairments, which is contradicted by Trewin et al. [16]. Our findings support Trewin et al.'s conclusion that swiping is accessible: relative to other tasks, steering (the closest of our tasks to swiping, and arguably more difficult) was not disproportionately hard for users with motor impairments compared to those without.

While spurious touches were a non-issue for participants without motor impairments, they were common for many users with motor impairments. Future work should investigate why spurious touches are a problem for some people with motor impairments and not others, as well as the extent to which these touches interfere with more realistic tasks that include multiple on-screen targets and where multitouch gestures are also available. For users with a high rate of spurious touches (e.g., above $40 \%$ of trials) the ability to disable multitouch input may be useful.

Finally, our participants without motor impairments were much younger than those with motor impairments. While an age-matched study would better isolate the effects of motor impairment from general age-related effects, our control group likely offers a baseline of near-optimal human input performance. Moreover, our findings contrast work showing that older adults are both faster and more accurate with the touchscreen than the mouse [2]. This contrast emphasizes the influence of motor impairment in our study and the importance of considering these two user groups distinct.

\section{ACKNOWLEDGMENTS}

This project was funded by NSF grant IIS-1350438. 


\section{REFERENCES}

1. Lisa Anthony, YooJin Kim, and Leah Findlater. 2013. Analyzing user-generated youtube videos to understand touchscreen use by people with motor impairments. In Proceedings of the SIGCHI Conference on Human Factors in Computing Systems (CHI '13), 1223-1232. http://doi.org/10.1145/2470654.2466158

2. Leah Findlater, Jon E. Froehlich, Kays Fattal, Jacob O. Wobbrock, and Tanya Dastyar. 2013. Age-related differences in performance with touchscreens compared to traditional mouse input. In Proceedings of the SIGCHI Conference on Human Factors in Computing Systems (CHI '13), 343-346. http://doi.org/10.1145/2470654.2470703

3. Tiago Guerreiro, Hugo Nicolau, Joaquim Jorge, and Daniel Gonçalves. 2010. Towards accessible touch interfaces. In Proceedings of the ACM SIGACCESS Conference on Computers and Accessibility (ASSETS'10), 19-26. http://doi.org/10.1145/1878803.1878809

4. Christian Holz and Patrick Baudisch. 2011. Understanding Touch. In Proceedings of the SIGCHI Conference on Human Factors in Computing Systems, 2501-2510. http://doi.org/10.1145/1978942.1979308

5. International Organization for Standardization. 2002. Ergonomic requirements for office work with visual display terminals (VDTs)-Requirements for nonkeyboard input devices.

6. Curt B Irwin and Mary E Sesto. 2012. Performance and touch characteristics of disabled and non-disabled participants during a reciprocal tapping task using touch screen technology. Applied ergonomics 43, 6: 1038-43. http://doi.org/10.1016/j.apergo.2012.03.003

7. Shaun K Kane, Chandrika Jayant, Jacob O Wobbrock, and Richard E Ladner. 2009. Freedom to roam: a study of mobile device adoption and accessibility for people with visual and motor disabilities. In Proceedings of the ACM SIGACCESS Conference on Computers and Accessibility (ASSETS'09), 115-122. http://doi.org/10.1145/1639642.1639663

8. I. Scott MacKenzie and Poika Isokoski. 2008. Fitts' throughput and the speed-accuracy tradeoff. In Proceedings of the SIGCHI Conference on Human Factors in Computing Systems (CHI'08), 1633-1636. http://doi.org/10.1145/1357054.1357308

9. Kyle Montague, Vicki L Hanson, and Andy Cobley. 2012. Designing for individuals: usable touch-screen interaction through shared user models. In Proceedings of the ACM SIGACCESS Conference on Computers and Accessibility (ASSETS'12), 151-158. http://doi.org/10.1145/2384916.2384943
10. Martez E Mott, Radu-Daniel Vatavu, Shaun K Kane, and Jacob O Wobbrock. 2016. Smart Touch: Improving Touch Accuracy for People with Motor Impairments with Template Matching. In Proceedings of the 2016 CHI Conference on Human Factors in Computing Systems, 1934-1946. http://doi.org/10.1145/2858036.2858390

11. Maia Naftali and Leah Findlater. 2014. Accessibility in Context: Understanding the Truly Mobile Experience of Smartphone Users with Motor Impairments. In Proceedings of the ACM SIGACCESS Conference on Computers and Accessibility (ASSETS '14), 209-216. http://doi.org/10.1145/2661334.2661372

12. Hugo Nicolau, Tiago Guerreiro, Joaquim Jorge, and Daniel Gonçalves. 2014. Mobile touchscreen user interfaces: Bridging the gap between motor-impaired and able-bodied users. Universal Access in the Information Society 13: 303-313. http://doi.org/10.1007/s10209-013-0320-5

13. Pekka Parhi, Amy K Karlson, and Benjamin B Bederson. 2006. Target size study for one-handed thumb use on small touchscreen devices. In Proceedings of MobileHCI '06, 203-210. http://doi.org/10.1145/1152215.1152260

14. M. E. Sesto, C. B. Irwin, K. B. Chen, A. O. Chourasia, and D. A. Wiegmann. 2012. Effect of Touch Screen Button Size and Spacing on Touch Characteristics of Users With and Without Disabilities. Human Factors: The Journal of the Human Factors and Ergonomics Society 54, 3: 425-436. http://doi.org/10.1177/0018720811433831

15. R. William Soukoreff and I. Scott MacKenzie. 2004. Towards a standard for pointing device evaluation, perspectives on 27 years of Fitts' law research in HCI. International Journal of Human-Computer Studies 61, 6: 751-789. http://doi.org/10.1016/j.ijhcs.2004.09.001

16. Shari Trewin, Cal Swart, and Donna Pettick. 2013. Physical accessibility of touchscreen smartphones. In Proceedings of the 15th International ACM SIGACCESS Conference on Computers and Accessibility (ASSETS '13), 1-8. http://doi.org/10.1145/2513383.2513446

17. Chat Wacharamanotham, Jan Hurtmanns, Alexander Mertens, Martin Kronenbuerger, Christopher Schlick, and Jan Borchers. 2011. Evaluating Swabbing: a touchscreen input method for elderly users with tremor. In Proceedings of the SIGCHI Conference on Human Factors in Computing Systems (CHI'11), 623-626. http://doi.org/10.1145/1978942.1979031

18. Jacob O Wobbrock, Leah Findlater, Darren Gergle, and James J Higgins. 2011. The Aligned Rank Transform for Nonparametric Factorial Analyses Using Only Anova Procedures. In Proceedings of the SIGCHI 
Conference on Human Factors in Computing Systems (CHI '11), 143-146.

http://doi.org/10.1145/1978942.1978963

19. Jacob O Wobbrock, Kristen Shinohara, and Alex Jansen. 2011. The effects of task dimensionality, endpoint deviation, throughput calculation, and experiment design on pointing measures and models. Proceedings of the 2011 annual conference on Human factors in computing systems (CHI'11): 1639. http://doi.org/10.1145/1978942.1979181

20. Yu Zhong, Astrid Weber, Casey Burkhardt, et al. 2015. Enhancing Android Accessibility for Users with Hand Tremor by Reducing Fine Pointing and Steady Tapping. In Proceedings of the 12th Web for All Conference, 29.

http://doi.org/10.1145/2745555.2747277 